\title{
Telocentrosomes organize telomere gathering
}

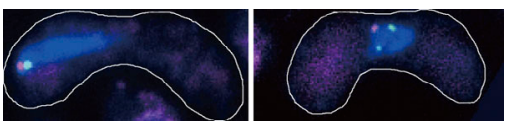

Telomeres (green) cluster at the spindle pole body (red) in a control cell (left) but keep their distance in a cell lacking the dynein light and heavy chains (right).

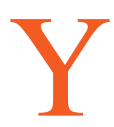

oshida et al. identify a new job for microtubule-organizing centers (MTOCs)_-helping telomeres huddle during meiosis.

The most famous

MTOC is the centrosome, a permanent structure that serves as a hub for the cell's microtubules and anchors the spindle during mitosis. But cells also deploy ephemeral MTOCs to perform other tasks. In fission yeast, for instance, part-time MTOCs help position the nucleus during mitosis.

Yoshida et al. uncovered a new MTOC function in fission yeast while studying the clustering of telomeres, the caps that protect chromosome ends. During meiosis, telomeres crowd together near the nuclear membrane, a maneuver that enables homologous chromosomes to pair. Previous studies suggested that dynein helps bring telomeres together, and the researchers found that kinesin motors also provide a push.

Because these molecular motors stick to microtubules, the team investigated how these filaments affect telomere movements. They discovered that a $\gamma$-tubulin-containing MTOC, which they dubbed the telocentrosome, formed near the telomeres and that its microtubules helped draw the chromosome caps together near the spindle pole body, the yeast equivalent of the centrosome. In cells lacking one protein necessary for telocentrosome formation, telomeres remained aloof, and homologous chromosomes didn't pair properly.

The researchers think that dynein and kinesin interconnect the microtubules linked to the spindle pole body and the telocentrosome, sliding along these fibers to help reel in the telomeres. Still unknown is what spurs the telocentrosome to form at a specific point in meiosis and what causes it to disassemble.

Yoshida, M., et al. 2013. J. Cell Biol. http://dx.doi.org/10.1083/jcb.201207168.

\section{SecYEG gets grabby with new proteins}

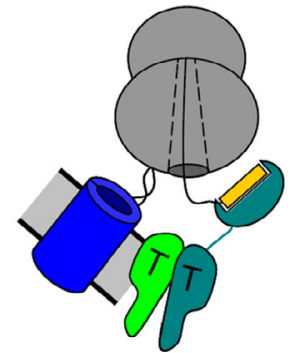

The ribosome (dark gray) and the rest of the targeting complex are about to hand over a protein to SecYEG (blue).

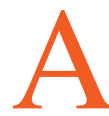
kopian et al. show that the bacterial protein channel Sec YEG - the homologue of eukaryotic Sec61-deforms its molecular partner to take custody of newly manufactured proteins.

SecYEG directs freshly synthesized proteins out of the cell or into the bacterial membrane. A ribosome carrying a nascent protein strand (together known as the RNC) meets up with the signal recognition particle (SRP), which then locates its receptor, FtsY, on the bacterial membrane. Together, this targeting complex delivers the nascent protein to SecYEG. Researchers didn't know whether SecYEG was a passive recipient or took a more active role in this handoff, reshaping the targeting complex so that it drops the nascent protein.

The SRP-FtsY combo flips among several states, including an early state and an active state that unloads its cargo and allows GTP hydrolysis. The RNC stabilizes the early state, presumably preventing premature cargo release. Akopian et al. found that SecYEG undermines the early conformation but stabilizes the active form of the complex. SecYEG sticks to the ribosome and joins the targeting complex, changing its conformation to activate cargo transfer and GTP hydrolysis.

Thus, instead of receiving the nascent protein, SecYEG grabs it along with the ribosome. Phospholipids also have a role in this process. They nudge SRP-FtsY away from the early state, but they can't overcome the countervailing effect of RNC and need help from SecYEG to dislodge the nascent protein strand.

Akopian, D., et al. 2013. J. Cell Biol. http://dx.doi.org/10.1083/jcb.201208045.

\section{Condensin II helps sisters find their identity}
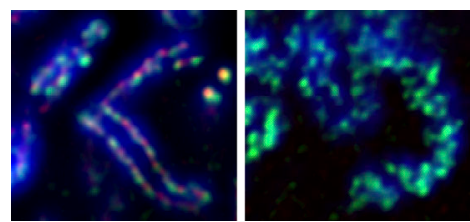

Late $\mathrm{S}$ phase sister chromatids (blue) are distinct in a control cell (left) but unresolved in a cell (right) missing a key portion of condensin II (red).

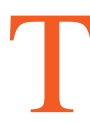

he condensin II complex gives cells a head start on mitosis by helping to separate sister chromatids during $\mathrm{S}$ phase, Ono et al. show.

Chromosomes are strung out and invisible for most of the cell cycle, not showing themselves until early in mitosis. But the process of chromosome condensation may begin earlier in the cell cycle. The protein complexes condensin I and condensin II help orchestrate chromosome condensation during mitosis. Unlike condensin I, which is trapped in the cytoplasm until prometaphase, condensin II dwells in the nucleus throughout interphase, raising the possibility that it acts before the onset of mitosis.
Ono et al. spurred cells to undergo premature chromosome condensation, in which the chromosomes compact and become visible before mitosis. G1 cells showed a single set of chromatids, but paired sister chromatids began to show up late in $\mathrm{S}$ phase and were clearly visible by G2. In cells lacking a component of condensin II, however, distinct chromatids rarely formed in $\mathrm{S}$ phase.

The team then used fluorescence in situ hybridization to tag and track matching locations on sister chromatids. The fluorescent signals from two sister chromatids were farther apart when cells carried condensin II than when they lacked a component of the complex, suggesting that condensin II helps each newly synthesized chromatid distance itself from its sister. The findings indicate that, during interphase, condensin II helps to separate sister chromatids shortly after DNA duplication, preparing them to condense and then segregate in the next mitosis. Ono, T., et al. 2013. J. Cell Biol. http://dx.doi.org/10.1083/jcb.201208008. 